\title{
New insights from unbiased panel and whole-exome sequencing in a large Chinese cohort with disorders of sex development
}

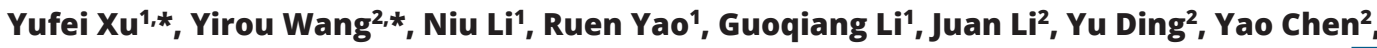 \\ Xiaodong Huang', Yuling Chen', Yanrong Qing', Tingting Yu' ${ }^{1}$, Yongnian Shen², \\ Xiumin Wang ${ }^{2}$, Yiping Shen ${ }^{3,4}$ and Jian Wang' \\ ${ }^{1}$ Department of Medical Genetics and Molecular Diagnostic Laboratory, ${ }^{2}$ Department of Endocrinology and \\ Metabolism, Shanghai Children's Medical Center, Shanghai Jiao Tong University School of Medicine, Shanghai, China, \\ ${ }^{3}$ Division of Genetics and Genomics, Boston Children's Hospital, Boston, Massachusetts, USA, ${ }^{4}$ Department of \\ Neurology, Harvard Medical School, Boston, Massachusetts, USA \\ *(Y Xu and $\mathrm{Y}$ Wang contributed equally to this work)
}

Correspondence should be addressed to $X$ Wang or $Y$ Shen or J Wang Email wangxiumin1019@126.com or Yiping.Shen@childrens. harvard.edu or labwangjian@shsmu.edu.cn

\begin{abstract}
Context: Diagnosis of non-chromosomal type disorders of sex development (DSD) has long been challenging. There is still no research on overview of a large Chinese DSD cohort.

Objective: To determine the etiologic diagnosis through unbiased large-scale panel sequencing and whole-exome sequencing (WES) within a large Chinese DSD cohort.

Design: Patients were recruited according to the inclusion criteria of DSD. The applied panel contains 2742 known disease-causing genes, including all known diagnostic genes for DSD.

Methods: Targeted panel sequencing (TPS) was performed, and identified candidate variants were verified. Variant pathogenicities were evaluated according to established guidelines. WES was performed for randomly selected negative samples.

Results: This study included 125 patients. Seventy-five variants were identified by TPS and 31 variants were reported for the first time. Pathogenic and likely pathogenic variants accounted for 38.7 and 30.7\%, respectively. On the basis of clinical certainty, etiologic diagnostic rates of 46.9 and $10.3 \%$ were obtained for $46, X Y$ and $46, X X$ DSD patients, respectively. We reported novel candidate genes (BMPR1B, GNAS, GHR) and regions of copy number variants outside the expected DSD genotype-phenotype correlation and determined a founder mutation (SRD5A2 p.R227Q) in patients with $5 \alpha$-reductase deficiency. Further WES in randomly selected negative samples identified only one among 14 negative samples as a variant of uncertain significance, indicating that WES did not improve the diagnostic rate. Conclusions: This is the first report of the applying unbiased TPS in a large Chinese cohort of patients with 46,XY and $46, X X$ DSD. Our findings expand the gene, mutation and phenotype spectra of the rare types of DSD in the Chinese population and provide new insight into the current understanding of the etiologies of DSD.
\end{abstract}

\section{Introduction}

Disorders of sex development (DSD) are a large group of heterogeneous diseases characterized by atypia of chromosomes, gonads or anatomical structures. The

established DSD classification, which was proposed by Lawson Wilkins Pediatric Endocrinology Society and European Pediatric Endocrinology Society at the Chicago 
Consensus Conference in 2006, comprise sex chromosome DSD, 46,XY DSD and 46,XX DSD according to the karyotype (1). On the basis of the underlying etiologies, DSD can be further divided into several subclasses, such as primary gonadal dysgenesis, deficient hormone synthesis or action, central hypogonadism and syndromic condition $(1,2)$. Epidemiological surveys show that approximately 2.2 in 10,000 newborns present with ambiguous genitalia $(3,4)$. The total incidence of DSD is supposed to be much higher than the above estimate, given that many associated DSD phenotypes like gynecomastia and primordial uterus might not be noticed until puberty (5). Numerous resulting problems, including gender uncertainty, genital malformation and infertility, cause profound physiological and psychological impact to the patients themselves and their families. Moreover, some types of DSD pose a risk of gonadal malignancy $(6,7)$. Current evidence indicates that numerous DSD pathogeneses are genetically determined, especially detrimental variants in genes involved in regulating sexual development $(5,8,9$, $10,11,12)$. Early identification of molecular causes is not only helpful to establish appropriate treatment and longterm follow-up for these patients but also contributes to individualized genetic counseling for their families (8).

Next-generation sequencing (NGS) greatly improves the efficiency of molecular diagnosis by virtue of its advantages of rapid and high-throughput detection of all genes or target gene regions in the human genome $(13,14)$. Compared with whole-exome sequencing (WES) and whole-genome sequencing (WGS), targeted panel sequencing (TPS), owing to its lower data processing and analysis costs, shorter detection cycle and greater sequencing depth, has gradually become the preferred method in clinical diagnosis in many countries where medical insurance does not yet cover genetic testing (14, 15). Recently, we published a study demonstrating that proband-only medical exome sequencing, targeting 2742 known disease-causing genes, could serve as a first-tier genetic diagnostic test for patients with a wide range of genetic conditions and without prior clinical diagnosis in China (16).

Heterogeneous clinical phenotypes and complex pathogenesis contribute to the low clinical diagnostic rate of non-chromosomal type DSD (i.e., 46,XY DSD and $46, \mathrm{XX}$ DSD); thus, the diagnosis of such patients has been a long-standing challenge $(2,5,8,11,17)$. Besides identifying a genetic cause, the diagnosis of DSD requires systematic and multidisciplinary collaboration, including assessment of the phenotypes and the endocrine and gonadal function (17). The diagnosis can only be confirmed if the clinical evaluation is consistent with the molecular findings. Several studies have applied panel sequencing in DSD patients to improve the diagnostic yield $(18,19,20,21,22,23,24,25)$. These panels usually only include the majority of known DSD-associated genes, and few studies have analyzed 46,XX DSD patients owing to the limited cases. Furthermore, till now, studies have recruited a relatively small number of Chinese DSD patients $(18,19,24)$, and no overview of Chinese $46, \mathrm{XY}$ DSD and 46,XX DSD patients is available.

Here, we report the application of unbiased panel sequencing in a cohort of Chinese patients with 46,XY DSD and 46,XX DSD. This panel contains 2742 known disease-causing genes, including all known diagnostic genes for DSD. We intended to identify the candidate pathogenic variants of a large DSD cohort and obtain etiologic diagnoses combined with clinical certainty; evaluate the diagnostic utility of unbiased, large-scale panel sequencing and WES; further enrich the gene, mutation and phenotype spectra of rare DSD; and present an overview of Chinese 46,XY DSD and 46,XX DSD patients.

\section{Subjects and methods}

\section{Subjects}

Patients referred to Shanghai Children's Medical Center for suspected DSD from December 2016 to December 2018 were enrolled for further investigation. Detailed medical history was recorded by professional clinicians. The preliminary diagnosis of DSD was based on clinical manifestations and laboratory and imaging examinations. The main inclusion criteria included ambiguous external genitalia; male-type external genitalia with bilateral cryptorchidism and/or micropenis and/or hypospadias; female-type external genitalia with clitoral enlargement and/or labia majoris fusion and/or intralabial/inguinal mass and/or inguinal hernia; masculinization; gonadal dysplasia; absent secondary sexual characteristics during puberty; primary amenorrhea; gynecomastia; gender inconsistency between gonad and chromosome; family history of DSD and so forth. Some patients might have been detected by microarray, fluorescence in situ hybridization or sequencing of $S R Y$ or some other gene before. Only patients with negative results were included in this cohort. All patients had undergone karyotyping to be excluded from sex chromosome DSD and be classified into established DSD subclasses. Due to the low mappability of reads caused by the presence of a 
pseudogene with high homology with the CYP21A2 gene, we excluded associated cases in this study.

Written informed consent for the genetic analysis was obtained from the patients or the patients' parents. A total of 125 unrelated patients were eligible and recruited to this cohort. This study was approved by the Ethics Committee of Shanghai Jiao Tong University School of Medicine.

\section{Targeted gene panel sequencing and variant validation}

The entire procedure was performed as described by $\mathrm{Hu}$ et al. (16). Briefly, $3 \mu$ g genomic DNA was extracted from the patients' peripheral blood sample and randomly sheared into $150-200 \mathrm{bp}$ fragments. The target exons and flanking intronic regions were captured by the inherited disease panel kit (cat No. 5190-7519; Agilent Technologies Inc.), including 2742 disease-causing genes. NGS was performed with HiSeq X Ten (Illumina). Sequencing quality was assessed, and reads were aligned to the GRCh37/hg19 human reference sequence. All acquired single-nucleotide variants (SNVs) were further annotated and filtered on the Ingenuity ${ }^{\circledR}$ Variant Analysis platform (Ingenuity Systems, Redwood City, CA, USA). Candidate variants were validated by Sanger sequencing with specific-designed primers. The DNA of patients' parents was also isolated and subjected to Sanger sequencing to confirm the origin of the candidate variants.

\section{Pathogenicity analysis of the validated variants}

We screened all the identified variants and categorized the pathogenicity of variants according to the criteria recommended by the American College of Medical Genetics and Genomics guideline (26), which was further refined and evolved by the ClinGen Sequence Variant Interpretation Working Group (https://www. clinicalgenome.org/working-groups/sequence-variantinterpretation/) $(27,28)$. Copy number variants (CNVs) were analyzed using a combination of the current guideline (29) and in-house criteria of our laboratory. We analyzed the allele frequencies of the detected variants and checked whether the variants had been reported before by retrieving relevant information from population databases such as the Genome Aggregation Database (gnomAD) (http://gnomad.broadinstitute.org/), Exome Variant Server (http://evs.gs.washington.edu/ EVS/), 1000 Genome Project (http://www.1000genomes. org/), dbSNP (http://www.ncbi.nlm.nih.gov/snp) and disease databases such as HGMD (http://www.hgmd.cf.ac. uk/ac/index.php) and ClinVar (http://www.ncbi.nlm.nih. gov/clinvar). We assessed the evolutionary conservatism of the associated loci on the basis of protein information in UniProt (http://www.uniprot.org) and Clustal Omega multi-species sequence alignment software (https:// www.ebi.ac.uk/Tools/msa/clustalo/). We used a variety of prediction tools such as PolyPhen2 (http://genetics. bwh.harvard.edu/pph2/) and PROVEAN (http://provean. jcvi.org/index.php) to predict the possible impact of the variants. Phenotypes summarized on OMIM (https:// omim.org/), literatures searched on PubMed (http:// www.ncbi.nlm.nih.gov/pubmed) and dosage sensitivity curation information provided in ClinGen (https:// www.ncbi.nlm.nih.gov/projects/dbvar/clingen/) were synthesized to further evaluate the related gene variants.

\section{Establishment of etiologic diagnostic rate}

All the candidate variants were further analyzed in combination with the clinical assessment results to determine the diagnostic yield. (i) When the variants of one patient were classified as variants of uncertain significance (VUS) and the clinical assessment also lacked specificity, it would be defined as an uncertain diagnosis. (ii) When the variants were classified as VUS, but the clinical assessment (including inheritance mode, patient's phenotype, hormone levels, gonadal histopathology and experimental therapy outcome) had a specific direct link to the same condition, it would be defined as a clinical diagnosis. (iii) When the variants were classified as pathogenic or likely pathogenic and were consistent with the clinical data, it would be defined as a molecular diagnosis. (iv) When the variants were classified as pathogenic or likely pathogenic but were inconsistent with the clinical data, or we were unsure about the correlation between the genotypes and phenotypes, it would remain an uncertain diagnosis. The definite diagnosis can only be confirmed if the clinical evaluation is undoubtedly consistent with the molecular findings. Thus, both the clinical diagnosis and molecular diagnosis contribute to the etiologic diagnostic yield.

\section{Further WES}

Among the negative samples, 14 cases were further subjected to WES as described by Wang et al. (30). The adapter-ligated library was prepared with the SureSelectXT Library Prep Kit (Agilent Technologies). We used a SureSelectXT Human All Exon Kit v6 (Agilent Technologies) to enrich coding exons and flanking 
intronic regions as the capture library. Sequencing was conducted on the Illumina HiSeq 2500 system (Illumina). The remaining analysis steps are basically similar to the previous procedures described in previous subsections.

\section{Results}

\section{Quality control of sequencing}

The quality of hybridization capture libraries met the requirement of sequencing. Sequencing data quality analysis is mainly based on the following parameters: aligned reads, reads on target, average coverage, region of interest and uniformity. In addition, the quality of sequencing was indirectly judged by the number and classification of SNVs compared with the controls. The results showed that aligned reads were $>99 \%$; reads on target, $>70 \%$; average coverage, around $150 \times$; $>20 \times$ region of interest, $>98 \%$; uniformity, $>91 \%$ and the difference value of total variants compared with the controls, within 300-550.

\section{Identification and validation of variants}

One hundred twenty-five samples were screened by Ingenuity software. The main filtering index and parameters included confinement for coding regions and splice sites, minor allele frequencies $<1 \%$, removal of reported synonymous and polymorphic SNVs and input of relevant clinical features such as 'gonadal development' and 'gonadal dysplasia'. All novel variants were further filtered against in-house databases. The highly suspected variants were all verified by Sanger sequencing and parental background. The main clinical features, karyotypes, identified variants and parental validation of positive cases are summarized in Table 1. Information of the remaining negative cases is provided in Supplementary Table 1 (see section on supplementary data given at the end of this article). Candidate variants were identified in 64 patients, accounting for $51.2 \%$ $(65 / 125)$ of all patients.

\section{Analysis and evaluation of pathogenicity}

Seventy-five candidate variants were evaluated according to standard criteria recommended by several guidelines (mentioned in Methods) $(26,27,29)$. The results of the pathogenicity classification are shown in Table 1 , and the details of the evidence level applied appropriately are provided in Supplementary Table 2. Among these 75 variants, $38.7 \%(29 / 75)$ were categorized into pathogenic variants; $30.7 \%(23 / 75)$, likely pathogenic variants and $30.7 \%(23 / 75)$, VUS. In the $46, \mathrm{XY}$ DSD cohort, $59.4 \%$ (57/96) individuals were found to carry candidate variants (Fig. 1A). The proportion of variants at different evidence levels showed that the pathogenic and likely pathogenic variants accounted for 37.3 and $34.3 \%$, respectively (Fig. 1A). The targeted gene panel proved less suited for those affected with 46,XX DSD. Only $24.1 \%$ (7/29) individuals were found to carry candidate variants (Fig. 1B). The pathogenic and likely pathogenic variants accounted for 50.0 and $0.0 \%$, respectively.

\section{Unexpected findings with syndromic DSD cases and novel candidate genes/phenotypes}

Patients 8096 and 9055, presenting with syndromic DSD, were identified with large fragment duplications in genomes (Table 1). Based on the wide range, gene coverage, and so forth, the CNVs could be categorized into pathogenic variants and VUS (Supplementary Table 2). However, these regions still lack evidence for triplosensitivity and phenotypic correlation. We also identified variants in genes (BMPR1B, GNAS, GHR) that are not known to be related to DSD carried by patients 2765 , 4881 and 6500 (Table 1). The three genes are associated with brachydactyly, Albright's hereditary osteodystrophy and Laron syndrome, respectively $(31,32,33)$. However, our patients presented with gonadal dysplasia as the main phenotype, instead of skeletal dysplasia, which is the typical symptom of the above conditions. Based on the type of variation, allele frequencies and other criteria, these variants could be classified into pathogenic or likely pathogenic groups. We assumed that these variants might contribute to our patients' phenotypes, and the three genes could possibly be novel candidate genes responsible for DSD. However, due to the lack of evidence for certainty, we still regarded these situations as cases of uncertain diagnosis despite the pathogenicity classification.

\section{Etiologic diagnostic yield}

According to the integrated analysis between molecular results and clinical validation, we further determined the etiologic diagnostic rate. We excluded the variants that were not definitely related to DSD (i.e., variants within BMPR1B, GNAS, and GHR, as well as CNVs), as we mentioned before. Although some variants like $L H C G R$ p.C514S and $A R$ p.A48S/p.G209E were classified VUS 


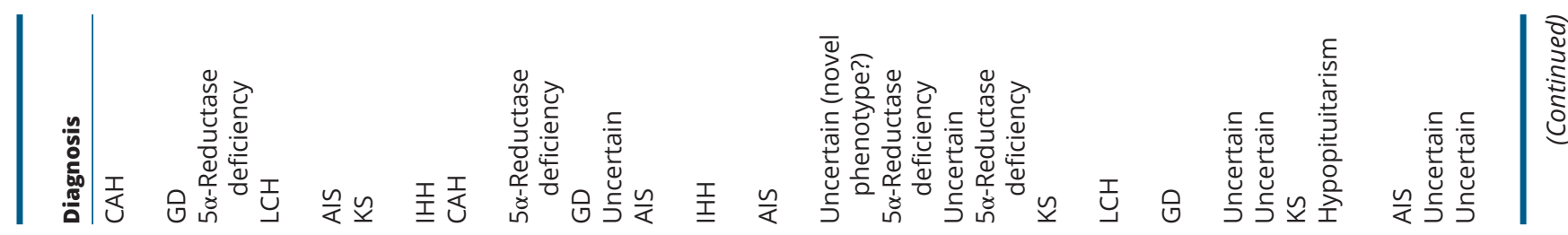

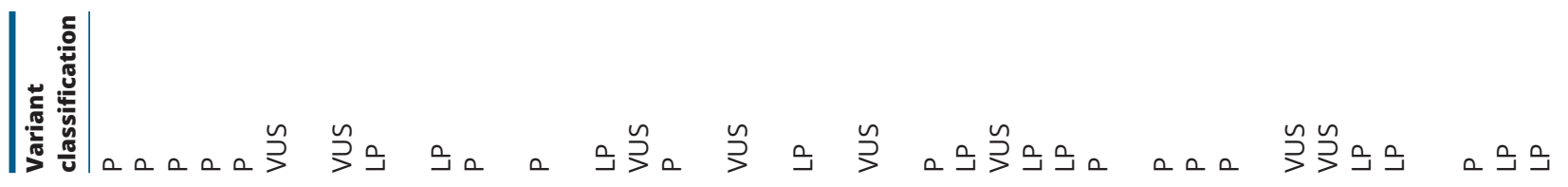

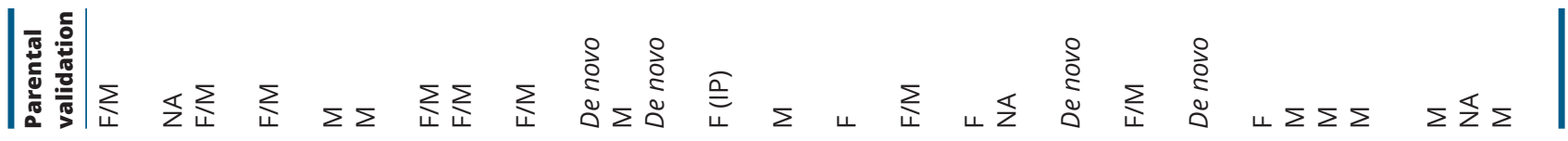
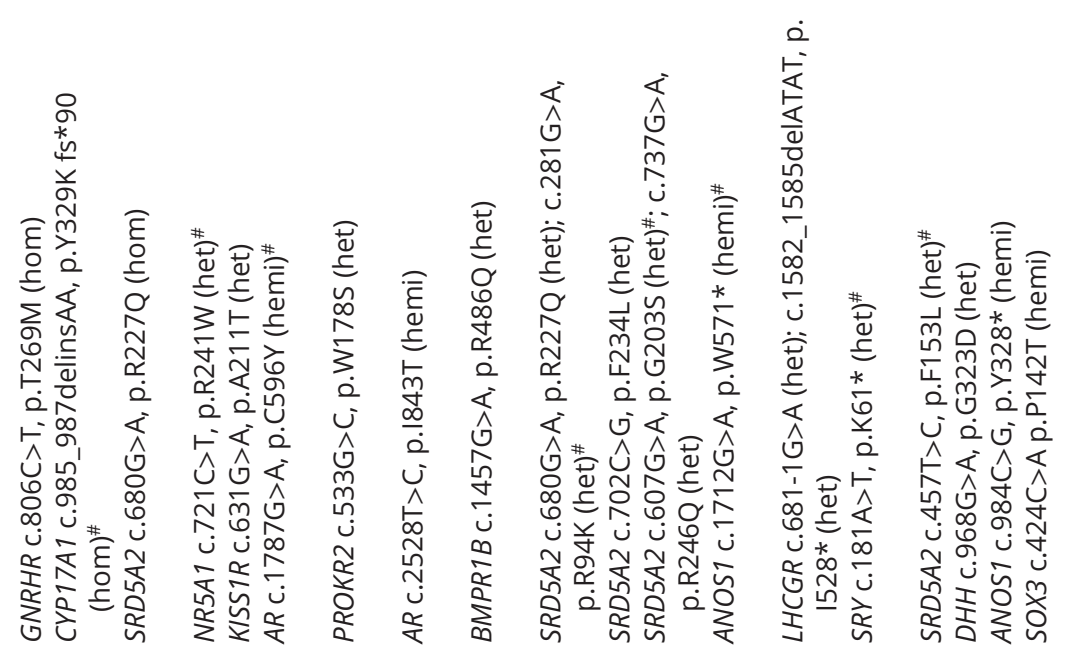

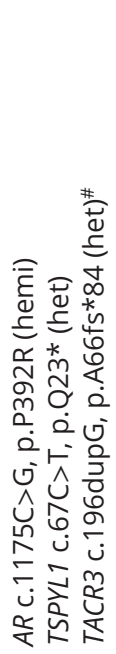

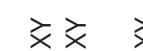

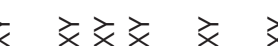

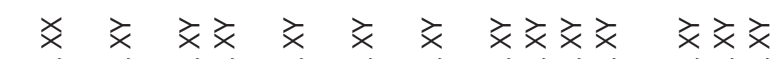

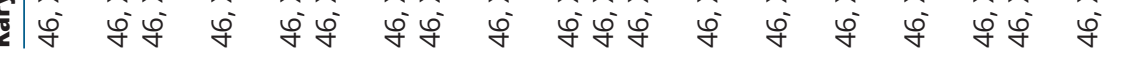
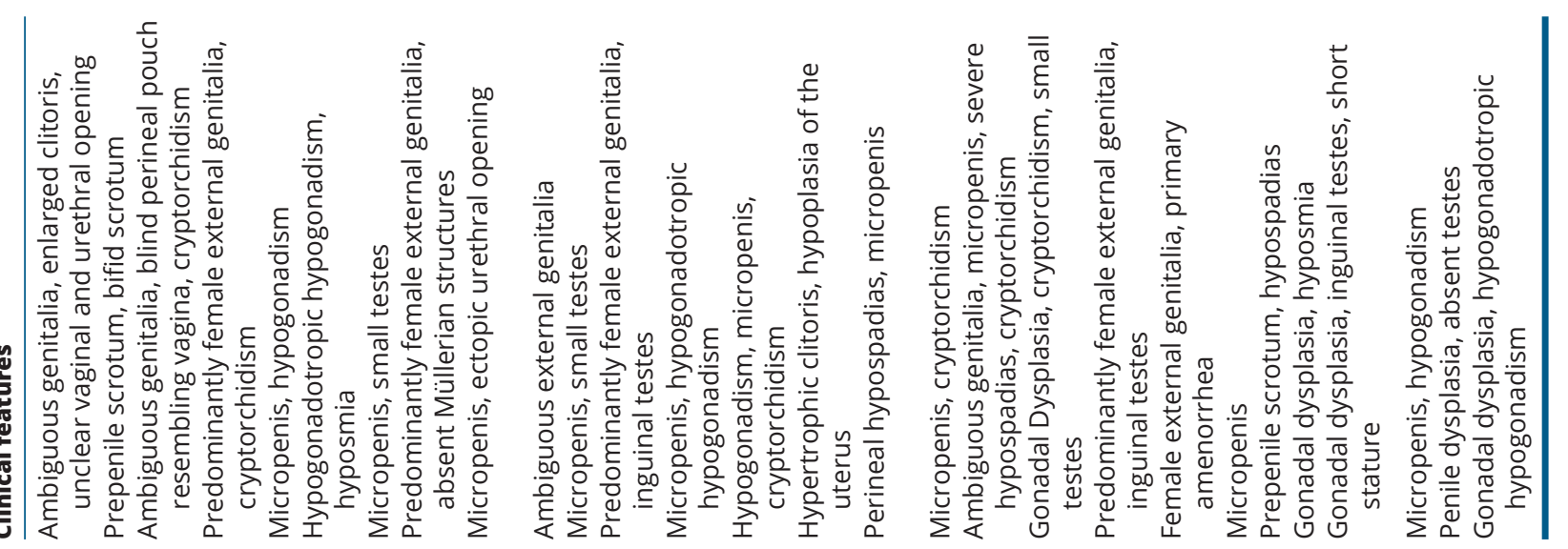

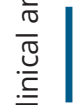

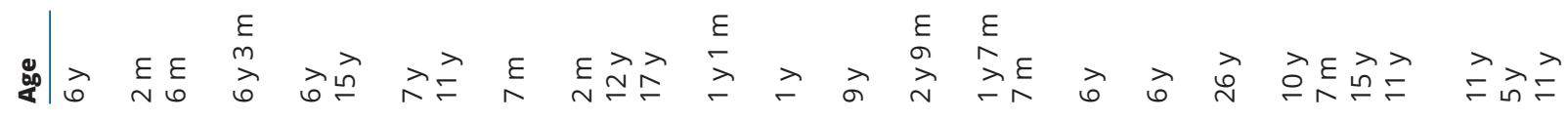




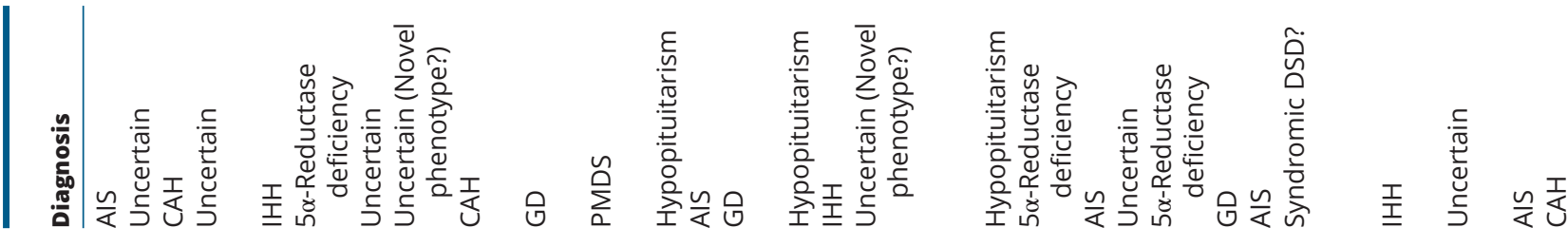

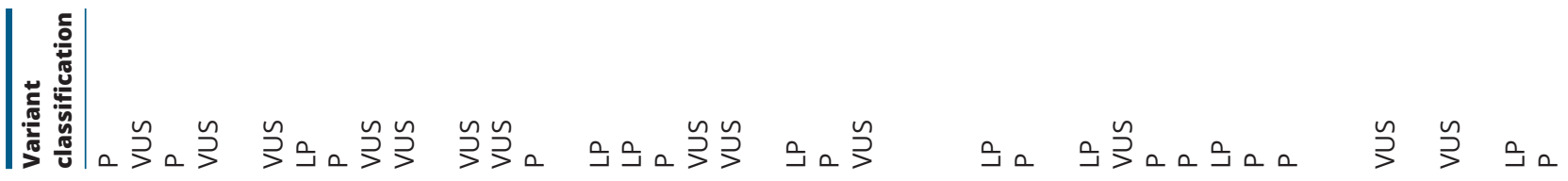

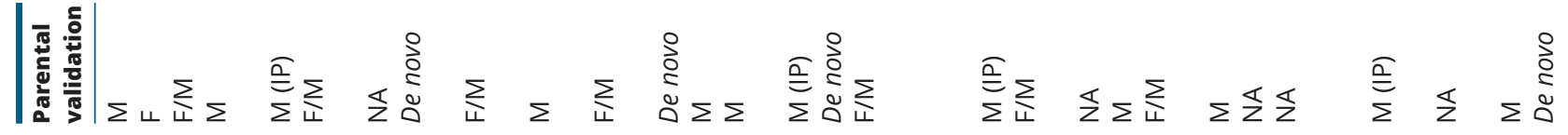

蓠

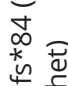

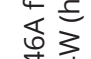

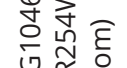

ن.

它运众

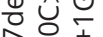

m

通定

논

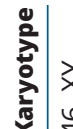

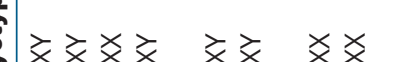

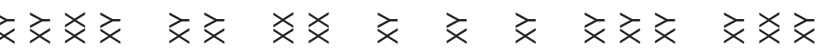

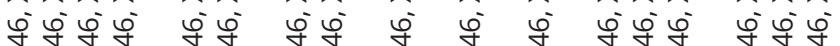

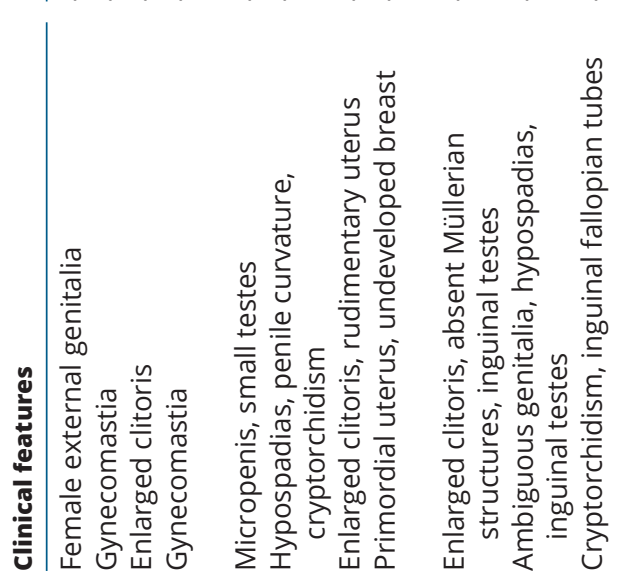

$$
\text { tr }
$$
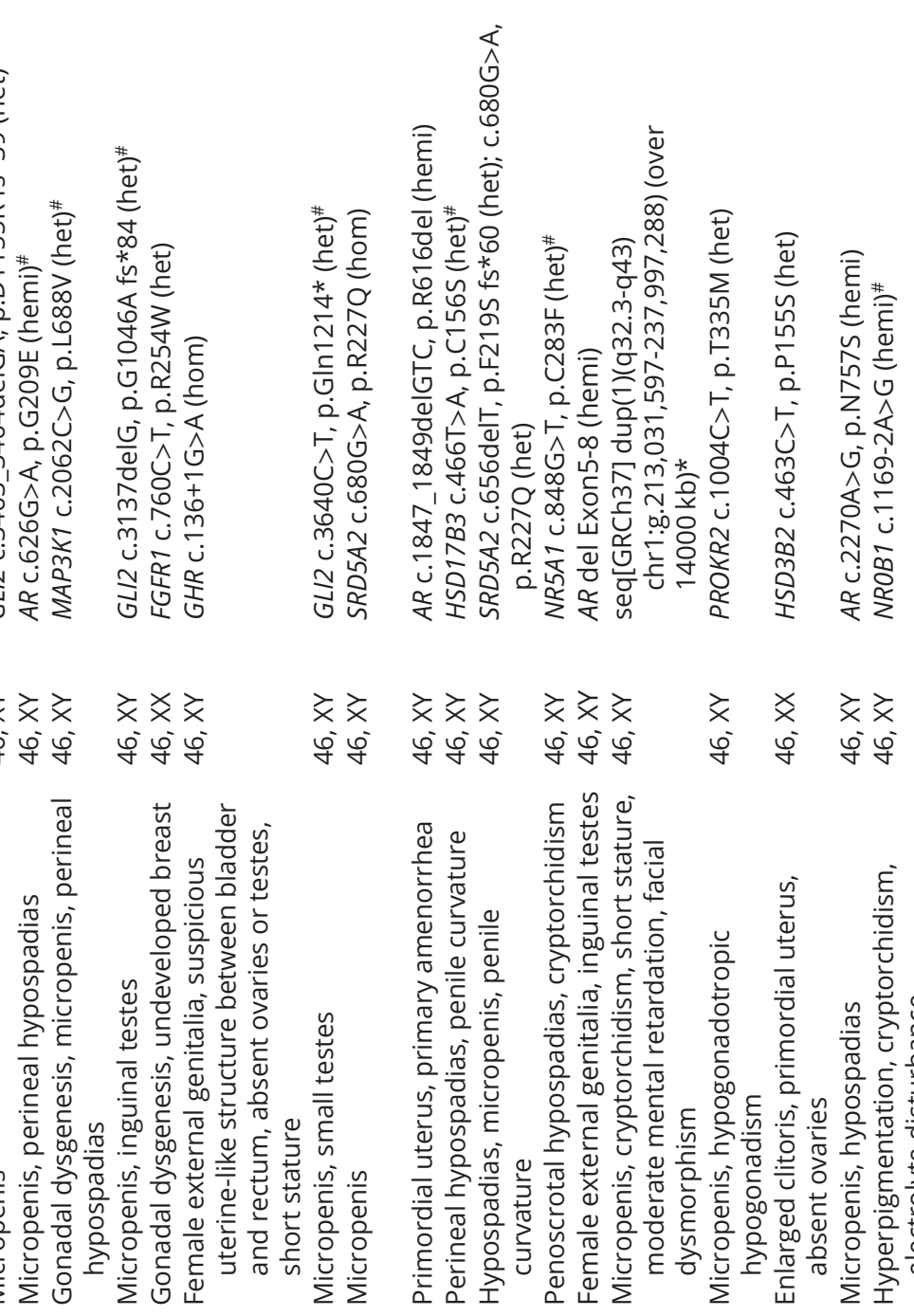


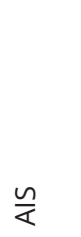

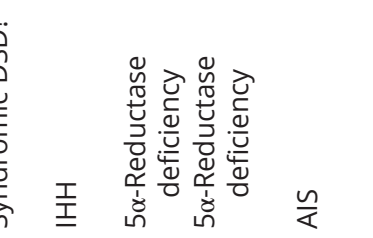

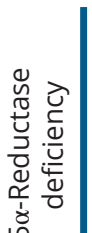

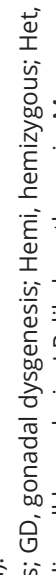

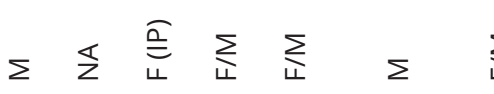

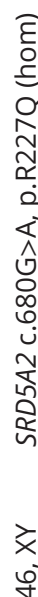

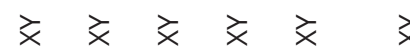

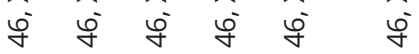

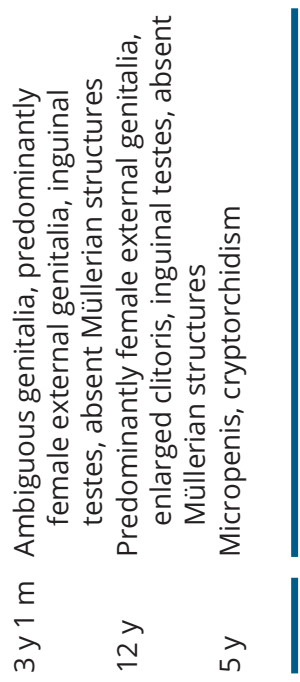

守

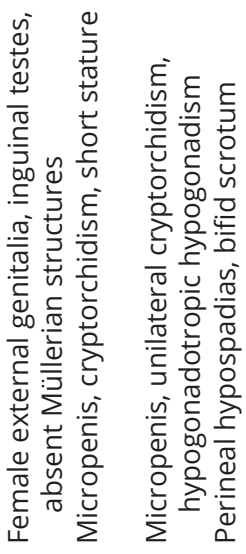

according to the current molecular evidence, these cases were included to calculate the final diagnostic yield on the basis of clinical certainty, which were confirmed by inheritance mode, phenotype, hormone levels, gonadal histopathology and enzyme assay, as needed. Accordingly, we obtained etiologic diagnostic rates of $46.9 \%$ for $46, \mathrm{XY}$ and $10.3 \%$ for $46, \mathrm{XX}$ DSD patients.

\section{Negative samples with further WES analysis}

Furthermore, we randomly selected 14 negative samples (six of 46,XY and eight of 46,XX) and performed WES additionally in an attempt to identify candidate genes that might have been missed by TPS. The re-sequencing sample IDs were 7902, 8017, 8135, 8145, 8318, 8510, 9056, 9262, 9524, 9553, 9743, 9771, 9954 and 9955 (Supplementary Table 1). The results showed only one patient (ID 9056) to be identified with a heterozygous variant (c.952dupA, p.Met318Asn fs*11) in the FAM111A gene, which may explain the symptoms of hypothyroidism, small thyroid gland, short stature and intrahepatic calcification. The correlation between phenotype of absent internal genitalia and the genotype remain to be clarified with more cases and experimental studies. In 13 other samples, we did not detect any variants responsible for the clinical manifestations.

\section{Overview of the present DSD patient cohort}

The 125 patients included in this study were divided into 76.8\% (96/125) 46,XY and 23.2\% (29/125) 46,XX patients according to the karyotype. (Fig. 2A). For patient age distribution, the number of patients in the puberty age group, namely $>9$ years old, was the largest (Fig. 2B). Identified variants were mainly distributed in 28 genes (Fig. 2C). Among all findings, 40.8\% (31/76) variants were novel. Variants in SRD5A2 and $A R$ genes were the most common ones, while others were relatively rare and sporadic. Notably, one variant (c.680G>A, p.R227Q) in the SRD5A2 gene was found in 75\% (9/12) patients with $5 \alpha$-reductase deficiency, indicating it is a hot-spot mutation among Chinese patients. Among the 46,XY DSD patients with etiologic diagnoses, the most common causes were disorders of androgen synthesis or action (DASA), accounting for 55.6\% (25/45), which mainly consisted of androgen insensitivity syndrome (AIS), caused by $A R$ gene variants, and steroid $5 \alpha$-reductase deficiency, caused by SRD5A2 gene variants (Fig. 3A). Congenital hypogonadotropic hypogonadism $(\mathrm{CHH})$ and gonadal dysgenesis (GD) accounted for $17.8 \%(8 / 25)$ and $15.6 \%(7 / 25)$, respectively. Other etiologies such as 
A

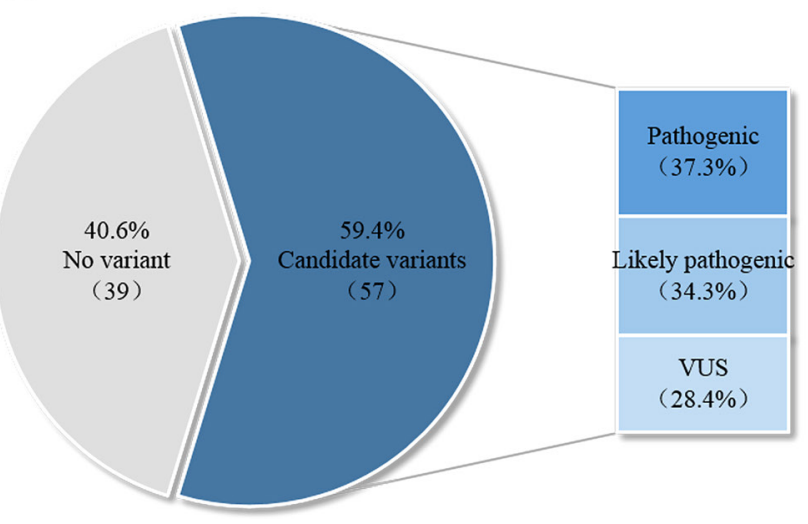

B

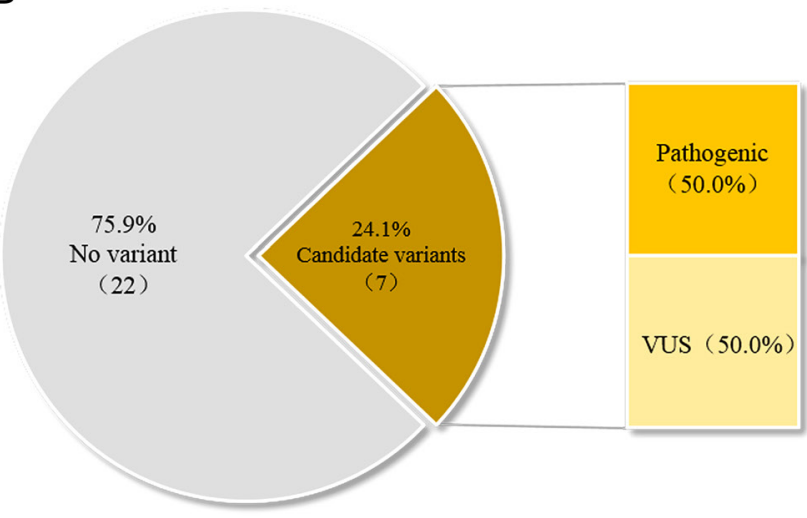

\section{Figure 1}

Positive rate of targeted panel sequencing and proportion of variants at different evidence levels. (A) In the 46,XY DSD cohort (96 patients), candidate variants were identified in 59.4\% (57/96) patients. The pathogenic variants, likely pathogenic variants and variants of uncertain significance (VUS) accounted for $37.3,34.3$ and $28.4 \%$ of the patients, respectively. (B) In the 46,XX DSD cohort (29 patients), candidate variants were identified in $24.1 \%(7 / 29)$ patients. The pathogenic variants and VUS accounted for 50.0 and $50.0 \%$, respectively.

persistent Müllerian duct syndrome and hypopituitarism accounted for $11.1 \%(5 / 25)$. In the 46 ,XX DSD patients with etiologic diagnoses, the main causes were $\mathrm{CAH}$ with 11ß-hydroxylase deficiency (2/3) and CHH (1/3). Within the remaining undiagnosed patients, the most common clinical manifestation was penile dysplasia (30/51) for 46, XY DSD and hypoplasia or absence of uterus (18/26) for 46,XX DSD (Fig. 3B).

\section{Discussion}

To our knowledge, this is the first study to apply unbiased NGS-based sequencing in a large Chinese cohort of patients with 46,XY and 46,XX DSD. In the past, before NGS was widely applied, only 13\% DSD patients had obtained an etiologic diagnosis (34). At present, NGS technologies such as WES and panel sequencing have been successfully applied in the diagnosis of DSD patients. Several studies have shown that the application of NGS improves the overall diagnostic rate of DSD, reaching $23-43 \%(18,19,20,21,22,23,24,25)$. Eggers et al. applied a DSD-related gene panel in a large-scale cohort study of foreign DSD patients, and the results showed that $43 \%$ of $46, \mathrm{XY}$ DSD patients received likely genetic diagnoses (23). The study also suggested that singletons and trios have no significant difference in the diagnostic rate of DSD patients (23). Thus far, few studies have applied WES
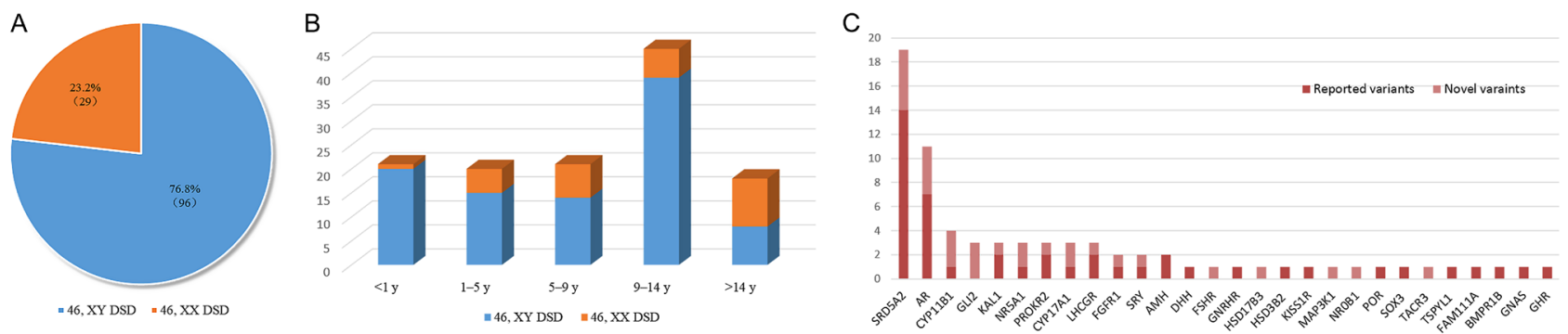

\section{Figure 2}

Distribution of the DSD cohort population and identified variants. (A) Ascertainment of the karyotype determined the major DSD classification. This study included 76.8\% (96/125) 46, XY and 23.2\% (29/125) 46, XX patients. (B) The age distribution of 46, XY and 46, XX DSD patients. (C) Distribution of the variants identified in DSD patients. Variants were identified in 28 genes. The total number of variants is shown for each gene, including previously reported variants and novel ones. 
A

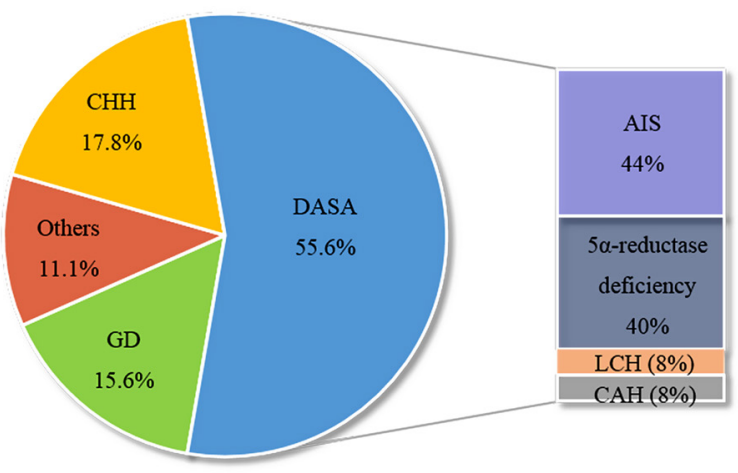

46, XY DSD patients with definite diagnosis

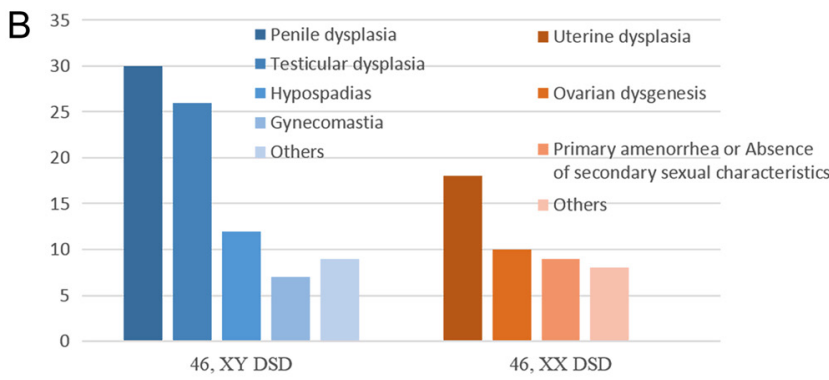

Main phenotypes of the undiagnosed patients

\section{Figure 3}

Characteristics of the diagnosed and undiagnosed DSD patients. (A) Proportion of different etiologies of 46,XY DSD patients with definite diagnoses in this study. The diagnoses have been categorized into subclasses based on the recommended classification of DSD (1). AIS, androgen insensitivity syndrome; $\mathrm{CAH}$, congenital adrenal cortical hyperplasia; $\mathrm{CHH}$, congenital hypogonadotropic hypogonadism; DASA, disorders of androgen synthesis or action; GD, gonadal dysgenesis; LCH, Leydig cell hypoplasia; (B) This bar graph shows the main clinical manifestations of undiagnosed patients. Abnormal development of external genitals was common in $46, \mathrm{XY}$ patients, and hypoplasia or absence of uterus or ovaries were the top characteristics of $46, X X$ patients.

in the diagnosis of DSD patients in large cohorts, and the diagnostic rate of WES lacks supporting evidence. In this study, we applied an unbiased and large-scale gene panel, targeted with 2742 known genetic disease-causing genes (containing all known DSD-associated genes), in a large Chinese 46,XY and 46,XX DSD patient cohort to identify candidate variants. Accordingly, we further integrated the analysis between molecular results and clinical validation to determine more credible and convincing etiologic diagnosis. It is noteworthy that CYP21A2, the most common CAH-causing gene (responsible for over 95\% CAH), was excluded in this study due to the existence of highly homologous pseudogenes, which makes specific capture by NGS difficult (35). Although this gene is included in our panel, we generally add single gene sequencing/multiplex ligation-dependent probe amplification tests for patients suspected with CAH. Thus, patients with CAH caused by CYP21A2 gene variants were not included in this study. Our results show that the etiologic diagnostic rate of $46, \mathrm{XY}$ DSD patients is slightly higher than that in other research, reaching $46.9 \%$. The diagnostic rate of 46,XX DSD patients is generally low, and here, we obtained an etiologic diagnostic rate of $10.3 \%$, which is similar to the results of other studies.

We identified 76 candidate variants, of which 31 are reported for the first time, further expanding the mutation spectrum of DSD. All identified variants were classified for pathogenicity strictly according to the recommended guidelines $(26,27,28,29)$. Variants of pathogenic and likely pathogenic classes accounted for $68.4 \%$. Nine variants including LHCGR p.C514S, AR p.A48S/p.G209E, CYP17A1 p.M262R/p.A398V and so forth could only be classified as VUS according to the current evidence. However, with the comprehensive verification of sequencing data quality, inheritance mode, clinical phenotypes, hormone levels, gonadal histopathology and enzyme assay, we found that the molecular findings were undoubtedly consistent with the clinical manifestation. Hence, it is reasonable to believe that these variants contribute to the phenotypes. In addition, we also found three genes that do not belong to the currently recognized pathogenic genes of DSD, namely GNAS, BMPR1B and GHR, but variants in these genes seemed to be deleterious. GNAS encodes the alpha subunit of the stimulatory guanine nucleotide-binding protein with a highly complex imprinting expression pattern. Pathogenic variants have been reported to cause resistance to parathyroid hormones and other hormones such as gonadotropin (36). Patients usually have multiple bone dysplasia as the main phenotype. A few patients may present with conditions associated with hormone level abnormalities, such as hypogonadism $(36,37)$. However, in this study, DSD was the main phenotype of patients with GNAS gene variants, and phenotypes such as primordial uterus were not reported. BMPR1B is a well-known osteopathy gene. As a receptor of bone morphogenetic protein, it participates in the regulation of growth and development through the TGF-beta signaling pathway (38). Thus far, no correlation between the BMPR1B gene and human gonadal development has been identified. However, animal model studies have found that its variation may affect the development of ovarian follicles 
(39). It has also been found that the GHR gene is expressed in granulosa cells and follicular membrane cells of the chicken ovary and plays an autocrine/paracrine regulatory role in follicular development (40). GHR overexpression has been found to increase difficulty in reproduction in zebrafish (41). Although the variants of these genes were presumed to be pathogenic according to the guidelines, their correlation with DSD phenotypes remains uncertain. The three genes are regarded as novel candidate DSDcausing genes. More cases and further functional studies are needed to define the correlation and pathogenicity.

CNVs have been reported to be identified in 33.3\% of DSD patients $(17,42)$, and although three-quarters were classified as being of uncertain clinical significance, upon review, half of these had in fact been reported in association with DSD (28). We identified large CNVs in patients with syndromic DSD. Combined with the records provided in Decipher database (https://decipher. sanger.ac.uk/), we found several case reports covering the chr1:213,031,597-237,997,288 region, which also manifested as abnormal genitalia. The other duplication within the chr22:36,649,117-38,380,539 region has not been reported yet. Genes involved in this region, like SOX10, may contribute to abnormal gonadal development, while triplosensitivity has not been confirmed yet (43). These findings lay the foundation for further study of the relationship between certain gene triplosensitivity and phenotypes.

Based on our own experience and the above doubts in the practical application of current variants classification guidelines, we believe that the guidelines play an important role in the judgment of variants' pathogenicity; however, they cannot be used as the only criterion for the definite diagnoses. In clinical practical application, comprehensive evaluation should be carried out in combination with clinical and published evidence to determine the etiologic diagnosis instead of mere classification of identified variants for DSD patients. In the light of the diagnostic approach flow recommended by European Cooperation in Science and Technology Action BM1303 DSDnet (17), we practiced the approach to diagnose this DSD cohort and obtained an etiologic diagnostic rate with certainty.

Although many studies, including ours, have confirmed that TPS greatly improved the diagnostic rate of DSD patients to a certain extent, there is still a large number of patients in whom no variant has been found, regardless of the scale of panel applied. We assumed that new, unknown genes involved in gonadal development may be missed by current panels. Therefore, we further performed WES in randomly selected negative samples in an attempt to identify candidate novel genes. Surprisingly, our results showed that only 1 of the 14 negative samples was identified with a suspected variant, which remains to be clarified in terms of the correlation between genotype and phenotype with further in-depth research. The remaining 13 samples remained undiagnosed, implying little difference in the diagnostic rate between TPS and WES, although according to recent recommendations, WES is a preferable tool for finding novel causes in undiagnosed DSD patients (17). Our findings deliver a new insight into the undiagnosed DSD patients. For negative cases initially screened by TPS, WES as the first choice for re-testing is not particularly suitable.

In addition to unknown novel genes, the following reasons might help explain the negative samples. First, non-coding variants in regulatory elements that alter gene expression contribute to the pathogenesis of abnormal gonadal development. Most research has mainly focused on the coding part of the genome, while some studies have found that variants in regulatory elements would affect the expression of target genes, resulting in spatiotemporal malfunction of DSD-related genes (44). These non-coding variants can be detected by WGS, which also has the ability to identify novel genes. Second, CNVs within small fragments that are not easily detected by the existing NGS technology could be missed, warranting re-analysis in combination with microarray $(44,45)$. Third, some key transcription factors such as SRY, SOX9 and FOXL2, and other genes are strictly regulated for specific spatiotemporal patterns in the process of sexual differentiation $(10,12$, 44). Somatic cell variants cannot be identified in DNA isolated by peripheral blood, while those in specific tissues such as gonads can be detected. Finally, the underlying pathogenesis of some cases may be multifactorial. The combination of epigenetic factors affecting early gonad development and environmental factors results in DSD phenotypes. Current genetic detection technology is not suitable for this situation $(5,17)$. Thus, in our subsequent studies, we will focus on aforementioned situation when we re-analyze these negative patients.

The pathogenesis involved in 46,XY DSD is better understood than that in $46, \mathrm{XX}$ DSD, largely because of the much larger number of patients and the much more easily detected gonadal malformations in the early stages; thus, the current diagnostic rate of panel sequencing tends to be much higher in 46,XY DSD patients (46, 47). This study of a Chinese DSD cohort yielded similar results. Our data showed that 46,XY DSD patients account for a large proportion in all age groups, especially in 
the group of 9 to 14 -year-olds. The etiologies of $46, \mathrm{XY}$ DSD are variable, including DASA, CHH, GD and other rare causes. AIS caused by $A R$ gene variants is the most frequent cause of $46, \mathrm{XY}$ DSD in the Chinese population. It was found that the risk of germ cell tumors in testicular dysplasia patients can be as high as $30 \%$, while that in AIS patients is about $15 \%$ (48). Therefore, it is particularly important for 46 , XY DSD patients to identify the etiology at an early stage. Moreover, a hot-spot variant (c.680G $>$ A, p.R227Q) in SRD5A2 gene is found in $75 \%$ patients with $5 \alpha$-reductase deficiency. Notably, this heterozygous variant was inherited from the unaffected parents, indicating that it accounts for a considerable proportion of the Chinese population. Combined with other research results of the SRD5A2 gene $(18,49,50)$, we suggested that it has a founder effect in the population of China. As for the remaining undiagnosed patients, penile or testicular dysplasia was most common manifestation, which may be somewhat related to certain nonspecific causes and associated reference range of measurements that has not been updated timely in China. Meanwhile, the number of $46, \mathrm{XX}$ DSD patients is lower due to the exclusion of a considerable number of $\mathrm{CAH}$ patients caused by CYP21A2 gene variants, mainly falling in the puberty group (over 9 years old) because phenotypes of 46, XX DSD such as primary amenorrhea and undeveloped secondary sexual characteristics were often unnoticed until puberty. In the 46 , XX DSD cohort, the most common etiology is CAH caused by CYP11B1 gene variants. Hypoplasia or absence of uterus and ovaries was the common in undiagnosed patients. The heterogeneity of imaging examination and the difficult assessment of ovarian function in childhood contribute to the low detection rate. Our results present a large number of non-chromosomal type DSD patients with various etiologies and provide strong support for further study of genotype and phenotype correlation in Chinese population.

In summary, this study applied unbiased TPS in a large Chinese cohort of patients with 46,XY and 46,XX DSD and further performed WES in randomly selected negative samples. With the current diagnosis flow and clinical certainty, we obtained etiologic diagnostic rates of $46.9 \%$ for $46, \mathrm{XY}$ and $10.3 \%$ for $46, \mathrm{XX}$ DSD patients. Re-analysis of patients undiagnosed by WES did not improve the diagnostic rate. We also provided an overview of the Chinese DSD population for the first time, reported novel candidate genes/regions outside the expected DSD genotype-phenotype correlation and determined a founder mutation in patients with $5 \alpha$-reductase deficiency. Our results expand the gene spectrum, mutation spectrum, and phenotypic spectrum of the rare types of DSD in the Chinese population; lay the foundation for further studies; and provide new insight into the current understanding of the underlying etiologies of DSD.

\section{Supplementary data}

This is linked to the online version of the paper at https://doi.org/10.1530/ EJE-19-0111.

\section{Declaration of interest}

The authors declare that there is no conflict of interest that could be perceived as prejudicing the impartiality of this study.

\section{Funding}

This research was supported by the National Natural Science Foundation of China (Grant No.81772303), 'Shuguang Program' supported by Shanghai Education Development Foundation and Shanghai Municipal Education Commission (Grant No. 18SG14), the Project of Shanghai Municipal Education Commission-Gaofeng Clinical Medicine (Grant No. 20152529) and the Doctoral Innovation Fund of Shanghai Jiao Tong University School of Medicine (Grant No. BXJ201949).

\section{Acknowledgements}

The authors would like to express sincere gratitude to all patients and their families for their participation in this study. They are grateful to the associated clinicians for providing detailed clinical information and for evaluating the clinical conformity after obtaining the molecular findings.

\section{References}

1 Hughes IA, Houk C, Ahmed SF, Lee PA \& Lawson Wilkins Pediatric Endocrine Society/European Society for Paediatric Endocrinology Consensus Group. Consensus statement on management of intersex disorders. Archives of Disease in Childhood 200691 554-563. (https:// doi.org/10.1136/adc.2006.098319)

2 Ostrer H. Disorders of sex development (DSDs): an update. Journal of Clinical Endocrinology and Metabolism 201499 1503-1509. (https:// doi.org/10.1210/jc.2013-3690)

3 Thyen U, Lanz K, Holterhus PM \& Hiort O. Epidemiology and initial management of ambiguous genitalia at birth in Germany. Hormone Research 200666 195-203. (https://doi.org/10.1159/000094782)

4 Lux A, Kropf S, Kleinemeier E, Jürgensen M, Thyen U \& DSD Network Working Group. Clinical evaluation study of the German network of disorders of sex development (DSD)/intersexuality: study design, description of the study population, and data quality. BMC Public Health 20099 110. (https://doi.org/10.1186/1471-2458-9-110)

5 Arboleda VA, Sandberg DE \& Vilain E. DSDs: genetics, underlying pathologies and psychosexual differentiation. Nature Reviews: Endocrinology 201410 603-615. (https://doi.org/10.1038/ nrendo.2014.130)

6 Cools M, Drop SL, Wolffenbuttel KP, Oosterhuis JW \& Looijenga LH. Germ cell tumors in the intersex gonad: old paths, new directions, moving frontiers. Endocrine Reviews 200627 468-484. (https://doi. org/10.1210/er.2006-0005)

7 Jørgensen A, Lindhardt Johansen M, Juul A, Skakkebaek NE, Main KM \& Rajpert-De Meyts E. Pathogenesis of germ cell neoplasia 
in testicular dysgenesis and disorders of sex development. Seminars in Cell and Developmental Biology 201545 124-137. (https://doi. org/10.1016/j.semcdb.2015.09.013)

8 Délot EC, Papp JC, DSD-TRN Genetics Workgroup, Sandberg DE, Vilain E \& Vilain E. Genetics of disorders of sex development: the DSD-TRN experience. Endocrinology and Metabolism Clinics of North America 201746 519-537. (https://doi.org/10.1016/j. ecl.2017.01.015)

9 Biason-Lauber A \& Chaboissier MC. Ovarian development and disease: the known and the unexpected. Seminars in Cell and Developmental Biology 201545 59-67. (https://doi.org/10.1016/j. semcdb.2015.10.021)

10 Wilhelm D, Palmer S \& Koopman P. Sex determination and gonadal development in mammals. Physiological Reviews 2007 87 1-28. (https://doi.org/10.1152/physrev.00009.2006)

11 Alhomaidah D, McGowan R \& Ahmed SF. The current state of diagnostic genetics for conditions affecting sex development. Clinical Genetics 201791 157-162. (https://doi.org/10.1111/cge.12912)

12 Bashamboo A \& McElreavey K. Human sex-determination and disorders of sex-development (DSD). Seminars in Cell and Developmental Biology 201545 77-83. (https://doi.org/10.1016/j. semcdb.2015.10.030)

13 Stark Z, Tan TY, Chong B, Brett GR, Yap P, Walsh M, Yeung A, Peters $\mathrm{H}$, Mordaunt $\mathrm{D}$, Cowie $\mathrm{S}$ et al. A prospective evaluation of whole-exome sequencing as a first-tier molecular test in infants with suspected monogenic disorders. Genetics in Medicine 201618 1090-1096. (https://doi.org/10.1038/gim.2016.1)

14 Xue Y, Ankala A, Wilcox WR \& Hegde MR. Solving the molecular diagnostic testing conundrum for Mendelian disorders in the era of next-generation sequencing: single-gene, gene panel, or exome/ genome sequencing. Genetics in Medicine 201517 444-451. (https:// doi.org/10.1038/gim.2014.122)

15 Yohe S, Hauge A, Bunjer K, Kemmer T, Bower M, Schomaker M, Onsongo G, Wilson J, Erdmann J, Zhou Y et al. Clinical validation of targeted next-generation sequencing for inherited disorders. Archives of Pathology and Laboratory Medicine 2015139 204-210. (https://doi. org/10.5858/arpa.2013-0625-OA)

$16 \mathrm{Hu}$ X, Li N, Xu Y, Li G, Yu T, Yao RE, Fu L, Wang J, Yin L, Yin Y et al. Proband-only medical exome sequencing as a cost-effective first-tier genetic diagnostic test for patients without prior molecular tests and clinical diagnosis in a developing country: the China experience. Genetics in Medicine 201820 1045-1053. (https://doi.org/10.1038/ $\operatorname{gim} .2017 .195)$

17 Audi L, Ahmed SF, Krone N, Cools M, McElreavey K, Holterhus PM, Greenfield A, Bashamboo A, Hiort O, Wudy SA et al. GENETICS IN ENDOCRINOLOGY: approaches to molecular genetic diagnosis in the management of differences/disorders of sex development (DSD): position paper of EU COST Action BM 1303 'DSDnet'. European Journal of Endocrinology 2018179 R197-R206. (https://doi. org/10.1530/EJE-18-0256)

18 Dong Y, Yi Y, Yao H, Yang Z, Hu H, Liu J, Gao C, Zhang M, Zhou L, Asan $e t$ al. Targeted next-generation sequencing identification of mutations in patients with disorders of sex development. BMC Medical Genetics 201617 23. (https://doi.org/10.1186/s12881-0160286-2)

19 Fan Y, Zhang X, Wang L, Wang R, Huang Z, Sun Y, Yao R, Huang X, Ye J, Han L et al. Diagnostic application of targeted nextgeneration sequencing of 80 genes associated with disorders of sexual development. Scientific Reports 20177 44536. (https://doi. org/10.1038/srep44536)

20 Baxter RM, Arboleda VA, Lee H, Barseghyan H, Adam MP, Fechner PY, Bargman R, Keegan C, Travers S, Schelley S et al. Exome sequencing for the diagnosis of $46, \mathrm{XY}$ disorders of sex development. Journal of Clinical Endocrinology and Metabolism 2015100 E333-E344. (https:// doi.org/10.1210/jc.2014-2605)
21 Kim JH, Kang E, Heo SH, Kim GH, Jang JH, Cho EH, Lee BH, Yoo HW $\&$ Choi JH. Diagnostic yield of targeted gene panel sequencing to identify the genetic etiology of disorders of sex development. Molecular and Cellular Endocrinology 2017444 19-25. (https://doi. org/10.1016/j.mce.2017.01.037)

22 Özen S, Onay H, Atik T, Solmaz AE, Özkınay F, Gökşen D \& Darcan Ş. Rapid molecular genetic diagnosis with next-generation sequencing in 46,XY disorders of sex development cases: efficiency and cost assessment. Hormone Research in Paediatrics 201787 81-87. (https:// doi.org/10.1159/000452995)

23 Eggers S, Sadedin S, van den Bergen JA, Robevska G, Ohnesorg T, Hewitt J, Lambeth L, Bouty A, Knarston IM, Tan TY et al. Disorders of sex development: insights from targeted gene sequencing of a large international patient cohort. Genome Biology 201617 243. (https:// doi.org/10.1186/s13059-016-1105-y)

24 Wang H, Zhang L, Wang N, Zhu H, Han B, Sun F, Yao H, Zhang Q, Zhu W, Cheng T et al. Next-generation sequencing reveals genetic landscape in 46, XY disorders of sexual development patients with variable phenotypes. Human Genetics 2018137 265-277. (https://doi. org/10.1007/s00439-018-1879-y)

25 Hughes LA, McKay Bounford K, Webb E, Dasani P, Clokie S, Chandran H, McCarthy L, Mohamed Z, Kirk JMW, Krone N et al. Next generation sequencing (NGS) to improve the diagnosis and management of patients with disorders of sex development (DSD). Endocrine Connections 20198 100-110. (https://doi.org/10.1530/ EC-18-0376)

26 Richards S, Aziz N, Bale S, Bick D, Das S, Gastier-Foster J, Grody WW, Hegde M, Lyon E, Spector E et al. Standards and guidelines for the interpretation of sequence variants: a joint consensus recommendation of the American College of Medical Genetics and Genomics and the Association for Molecular Pathology. Genetics in Medicine 201517 405-424. (https://doi.org/10.1038/gim.2015.30)

27 Biesecker LG, Harrison SM \& ClinGen Sequence Variant Interpretation Working Group. The ACMG/AMP reputable source criteria for the interpretation of sequence variants. Genetics in Medicine 201820 1687-1688. (https://doi.org/10.1038/gim.2018.42)

28 Abou Tayoun AN, Pesaran T, DiStefano MT, Oza A, Rehm HL, Biesecker LG, Harrison SM \& ClinGen Sequence Variant Interpretation Working Group. Recommendations for interpreting the loss of function PVS1 ACMG/AMP variant criterion. Human Mutation 201839 1517-1524. (https://doi.org/10.1002/ humu.23626)

29 Kearney HM, Thorland EC, Brown KK, Quintero-Rivera F, South ST \& Working Group of the American College of Medical Genetics Laboratory Quality Assurance Committee. American College of Medical Genetics standards and guidelines for interpretation and reporting of postnatal constitutional copy number variants. Genetics in Medicine 201113 680-685. (https://doi.org/10.1097/ GIM.0b013e3182217a3a)

30 Wang J, Yu T, Wang Z, Ohte S, Yao RE, Zheng Z, Geng J, Cai H, Ge Y, $\mathrm{Li} \mathrm{Y}$ et al. A new subtype of multiple synostoses syndrome is caused by a mutation in GDF6 that decreases its sensitivity to noggin and enhances its potency as a BMP signal. Journal of Bone and Mineral Research 201631 882-889. (https://doi.org/10.1002/jbmr.2761)

31 Lehmann K, Seemann P, Boergermann J, Morin G, Reif S, Knaus P \& Mundlos S. A novel R486Q mutation in BMPR1B resulting in either a brachydactyly type C/symphalangism-like phenotype or brachydactyly type A2. European Journal of Human Genetics $2006 \mathbf{1 4}$ 1248-1254. (https://doi.org/10.1038/sj.ejhg.5201708)

32 Ahrens W, Hiort O, Staedt P, Kirschner T, Marschke C \& Kruse K. Analysis of the GNAS1 gene in Albright's hereditary osteodystrophy. Journal of Clinical Endocrinology and Metabolism 200186 4630-4634. (https://doi.org/10.1210/jcem.86.10.7946)

33 Cogan JD, Ramel B, Lehto M, Phillips J 3rd, Prince M, Blizzard RM, de Ravel TJ, Brammert M \& Groop L. A recurring dominant negative 
mutation causes autosomal dominant growth hormone deficiency - a clinical research center study. Journal of Clinical Endocrinology and Metabolism 199580 3591-3595. (https://doi.org/10.1210/ jcem.80.12.8530604)

34 Arboleda VA, Lee H, Sánchez FJ, Délot EC, Sandberg DE, Grody WW, Nelson SF \& Vilain E. Targeted massively parallel sequencing provides comprehensive genetic diagnosis for patients with disorders of sex development. Clinical Genetics 201383 35-43. (https://doi. org/10.1111/j.1399-0004.2012.01879.x)

35 Lee HH. Mutational analysis of CYP21A2 gene and CYP21A1P pseudogene: long-range PCR on genomic DNA. Methods in Molecular Biology 20141167 275-287. (https://doi.org/10.1007/978-1-49390835-6_19)

36 Mantovani G \& Spada A. Mutations in the Gs alpha gene causing hormone resistance. Best Practice and Research: Clinical Endocrinology and Metabolism 200620 501-513. (https://doi.org/10.1016/j. beem.2006.09.001)

37 Agopiantz M, Journeau P, Lebon-Labich B, Sorlin A, Cuny T, Weryha G \& Leheup B. McCune-Albright syndrome, natural history and multidisciplinary management in a series of 14 pediatric cases. Annales d'Endocrinologie 201677 7-13. (https://doi.org/10.1016/j. ando.2016.01.002)

38 Laird M, Thomson K, Fenwick M, Mora J, Franks S \& Hardy K. Androgen stimulates growth of mouse preantral follicles in vitro: interaction with follicle-stimulating hormone and with growth factors of the TGF $\beta$ superfamily. Endocrinology 2017158 920-935. (https://doi.org/10.1210/en.2016-1538)

39 Al-Samerria S, Al-Ali I, McFarlane JR \& Almahbobi G. The impact of passive immunisation against BMPRIB and BMP4 on follicle development and ovulation in mice. Reproduction 2015149 403-411. (https://doi.org/10.1530/REP-14-0451)

40 Ahumada-Solórzano SM, Martínez-Moreno CG, Carranza M, ÁvilaMendoza J, Luna-Acosta JL, Harvey S, Luna M \& Arámburo C. Autocrine/paracrine proliferative effect of ovarian GH and IGF-I in chicken granulosa cell cultures. General and Comparative Endocrinology 2016234 47-56. (https://doi.org/10.1016/j.ygcen.2016.05.008)

41 Silva AC, Almeida DV, Nornberg BF, Pereira JR, Pires DM, Corcini CD, Junior AS \& Marins LF. Reproductive parameters of double transgenic zebrafish (Danio rerio) males overexpressing both the growth hormone (GH) and its receptor (GHR). Transgenic
Research 201726 123-134. (https://doi.org/10.1007/s11248-0169990-0)

42 Zargari M, Sadeghi MR, Shahhosseiny MH, Kamali K, Saliminejad K, Esmaeilzadeh A \& Khorshid HR. Fetal sex determination using non-invasive method of cell-free fetal DNA in maternal plasma of pregnant women during 6th-10th weeks of gestation. Avicenna Journal of Medical Biotechnology 20113 201-206.

43 Bondurand N, Dastot-Le Moal F, Stanchina L, Collot N, Baral V, Marlin S, Attie-Bitach T, Giurgea I, Skopinski L, Reardon W et al. Deletions at the SOX10 gene locus cause Waardenburg syndrome types 2 and 4. American Journal of Human Genetics $2007 \mathbf{8 1}$ 1169-1185. (https://doi.org/10.1086/522090)

44 Baetens D, Mendonça BB, Verdin H, Cools M \& De Baere E. Noncoding variation in disorders of sex development. Clinical Genetics 201791 163-172. (https://doi.org/10.1111/cge.12911)

45 de Ligt J, Boone PM, Pfundt R, Vissers LE, Richmond T, Geoghegan J, O'Moore K, de Leeuw N, Shaw C, Brunner HG et al. Detection of clinically relevant copy number variants with whole-exome sequencing. Human Mutation 201334 1439-1448. (https://doi. org/10.1002/humu.22387)

46 Buonocore F \& Achermann JC. Human sex development: targeted technologies to improve diagnosis. Genome Biology 201617257. (https://doi.org/10.1186/s13059-016-1128-4)

47 Hiort $\mathrm{O}$. The differential role of androgens in early human sex development. BMC Medicine 201311 152. (https://doi. org/10.1186/1741-7015-11-152)

48 Pleskacova J, Hersmus R, Oosterhuis JW, Setyawati BA, Faradz SM, Cools M, Wolffenbuttel KP, Lebl J, Drop SL \& Looijenga LH. Tumor risk in disorders of sex development. Sexual Development 20104 259-269. (https://doi.org/10.1159/000314536)

49 Cheng J, Lin R, Zhang W, Liu G, Sheng H, Li X, Zhou Z, Mao X $\&$ Liu L. Phenotype and molecular characteristics in 45 Chinese children with $5 \alpha$-reductase type 2 deficiency from South China. Clinical Endocrinology 201583 518-526. (https://doi.org/10.1111/ cen.12799)

50 Yuan S, Meng L, Zhang Y, Tu C, Du J, Li W, Liang P, Lu G \& Tan YQ. Genotype-phenotype correlation and identification of two novel SRD5A2 mutations in 33 Chinese patients with hypospadias. Steroids 2017125 61-66. (https://doi.org/10.1016/j. steroids.2017.06.010)

Received 17 February 2019

Revised version received 2 June 2019

Accepted 5 July 2019 